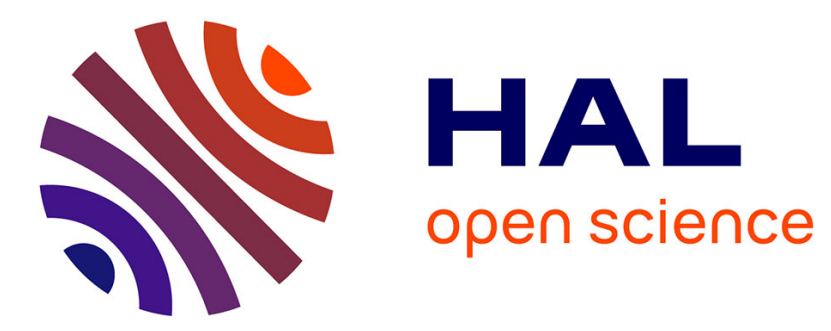

\title{
Un en-tête de décret attique conservé au Musée de Laon Jean-Yves Marc
}

\section{To cite this version:}

Jean-Yves Marc. Un en-tête de décret attique conservé au Musée de Laon. Revue des études anciennes, 1993, 95 (1-2), pp.143-156. halshs-00009876

\section{HAL Id: halshs-00009876 https://shs.hal.science/halshs-00009876}

Submitted on 31 Mar 2006

HAL is a multi-disciplinary open access archive for the deposit and dissemination of scientific research documents, whether they are published or not. The documents may come from teaching and research institutions in France or abroad, or from public or private research centers.
L'archive ouverte pluridisciplinaire HAL, est destinée au dépôt et à la diffusion de documents scientifiques de niveau recherche, publiés ou non, émanant des établissements d'enseignement et de recherche français ou étrangers, des laboratoires publics ou privés. 
Un en-tête de décret attique conservé au Musée de Laon.

Article paru dans « Revue des Etudes Anciennes » Tome 95, 1993 


\title{
UN EN-TÊTE DE DÉCRET ATTIQUE CONSERVÉ AU MUSÉE DE LAON*
}

\author{
Jean-Yves MARC ${ }^{* *}$
}

Résumé. - Le Musée de Laon conserve la moitié supéricure gauche d'un en-tête de décret attique inédit, sculpté dans les années 340/330 av. J.-C., où l'on reconnaît Athéna s'appuyant sur sa lance et retenant son bouclier posé à terre. Seul le début des deux premières lignes de l'inscription est aujourd'hui lisible.

L'analyse de l'iconographie du relief, puis du formulaire de l'inscription, montre que ces monuments n'étaient pas des documents figurant une représentation officielle de la divinité civique et reproduisant fidèlement un texte officiel.

Abstract. - The left part of an unknown attic document relief of the years 340/330 B.C., where we can see Athena with her weapons, is exposed in the muscum of Laon. Only the beginning of the two first lines can be read.

The iconography of this relief and the formulation of the inscription show that these monuments were not state documents with an official image of the civic goddess and the text voted by the assembly.

Deux synthèses ont été consacrées ces dernières années aux en-tête à relief. Ces deux études ${ }^{1}$ n'ont pu prendre en compte ni le relief trouvé très récemment à Delphes et publié

* Je remercie très chaleurcusement Madame Caroline Jorrand, conservatrice au Musée de Laon, qui m'a reçu cordialement à plusieurs reprises et s'est toujours efforcéc de faciliter mon travail en me communiquant généreusement archives et photographies. En dehors des fig. 1 et 2, photographies de l'auteur (négatifs EFA n L. 9875, 6a et L. 9875 , 8a), les illustrations ont été fournies pour la fig. 3 par l'Institut archéologique allemand d'Athènes (négatif $n^{\circ} 3884$ ), pour les fig. 4 et 7 par l'École française d'Athènes (clichés P. Collet, négatif $n^{\circ} 44465$ et cliché E. Séraf, négatif $n^{\circ} 66772$ ), pour la fig. 5 par le Musée Calvet (négatif n E.28) et pour la fig. 6 par l'ícole américaine d'études classiques à Athènes, fouilles de l'Agora (cliché C. A. Mauzy, négatif $n^{\circ}$ XXXVII-60).

** École française d'Athènes.

1. Carol L. Lawton, Attic Document Relief of the Classical and Hellenistic Periods: Their History, Development and Use, Diss. Princeton, 1984 ; Marion Miyter, Die griechischen Urkundenreliefs, AM, Beiheft 13 (1989), cf. le compte rendu de M. SÉve, Bull.Epigr., 1990, 136. I. KASPER-BUTZ a repris l'étude de ces documents dans une étude sur la représentation d'Athéna à Athènes et ses significations politiques : Die Göttin Athena im klassischen Athen. Athena als Repräsentantin des demokratischen Staates, Francfort, 1990.

REA, 95, 1993, nº 1-2, p. 143 à 156. 
par J.Ch. Moretti², ni celui qui est depuis peu exposé au Musée Bénaki à Athènes ${ }^{3}$. Il faut également ajouter aux reliefs qui y sont répertoriés ${ }^{4}$ la moitié supérieure gauche d'une stèle en marbre conservée au musée de Laon ${ }^{5}$, sur laquelle M. Marcadé avait attiré mon attention en 1983 (Fig.1). Cette stèle, de forme légèrement pyramidante, comportait un fronton, une vignette sculptée et une inscription dont n'est conservé que le début des deux premières lignes ${ }^{6}$.

2. Jean-Charles MorktTi, "Une vignette de traité à Delphes », $B C H 111,1987$, p. 157-166.

3. O. PÂnagia, "A New Record Relief in the Benaki Museum ", BICS 37, 1990, p. 135 à 141 et pl. 8 à 10.

4. M. Meyer, op. cit., a recensé deux cent quatorıe reliefs. On peut ajouter à son catalogue les dix relicfs suivant

Athènes, Mus. Acrop. inv. 2428. Cf. O. WALTER, Beschreibung der Reliefs im kleinen Akropolismuseum in Athen, Vienne, 1923, n² 25, p. 19-20 ; I. KASPI:R-BUTZ, op. cit., K 12, p. 228.

Athènes, Mus. Nat. inv. 2799. Cf. J. N. Svoronos, Das Athener Nationalmuseum, Athènes, 1937, n 450, p. 669 et pl. 223 ; I. KASPER-BUTZ, op. cit., K 22, p. 232.

Athènes, Mus. nat. inv. 2786. Cf. J. N. Svoronos, op. cit., n 450, p. 669 et pl. 223 ; I. KaSPER-BUTZ, op. cit., K 29, p. 234.

- Athènes, Mus. Acrop. inv. 2433. Cf. O. Walter, op. cit., nº 22, p. 19 ; I. KASPER-BUTZ, op. cit., K 31, p. 235. p. 235 .

- Athènes, Mus. Acrop. inv. $2439+2967$. Cf. O. WAlter, op. cit., ${ }^{\circ} 28$, p. 31 ; I. KASPER-BUTZ, op. cit., K 32 p. 235

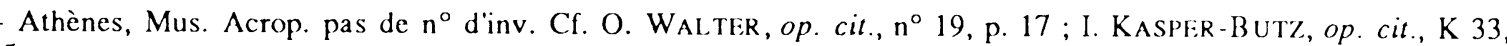

- Athènes, Mus. Acrop. inv. 4689. Cf. O. Wal.TER, op. cit., n² 20, p. 18 ; I. KaSPER-BUTz, op. cit., K 34, p. 235.

- Athènes, Mus. Acrop. inv. 2539. Cf. O. Walter, op. cit., n² 27, p. 20 ; I. KASPER-BuTz, op. cit., K 36, p. 236.

- Athènes, Mus. Acrop. inv. 2427. Cf. O. Walter, op. cit., nº 24, p. 19 ; I. KasPer-Butz, op. cit., K 37, p. 236.

- Athènes, Mus Acrop. inv. $3367+2542+$ Mus. Epigr. inv. 8024. Cf. O. WALTER, op. cit., n ${ }^{\circ} 55$, p. 38 et J. N. SVORONOS, op. cit., $\mathrm{n}^{\circ} 442$, p. 667 et pl. $217,2$.

Je pense en revanche qu'il n'y a aucune raison précise pour ajouter le relief Mus. Acrop. inv. 2432 qu'O. Walter range parmi les reliefs votifs (op. cit., $\mathrm{n}^{\circ} 47$, p. 33) mais qu'I. Kasper-Butz classe dans le genre des en-tête de décret (op. cit., K 27, p. 233-234).

5. Le relief provient d'une collection d'antiquités, réunie au début du siècle par Joseph Eugène Paul Marguerite de la Charlonie et exposée au Musée Municipal de Laon depuis 1955. Séduit par l'Antiquité grecque au cours d'une croisière en mer Egée en 1898, philhéllène convaincu, P. Marguerite de la Charlonie (18 avril 1844 - 26 octobre 1921), ingénieur des Arts et Manufactures et industriel, rassembla jusqu'à sa mort une importante collection d'Antiques, comportant 1700 pièces, vases, figurines en terre cuite, sculptures en marbre, achetées à l'occasion de nombreux voyages, au moins une douzaine, en Méditerranée (Égypte, Turquie, Grèce, Italie) et de ventes en France. Conseillé par son ami Edmond Pottier, dont il a suivi assidument les cours à l'École du Louvre, ainsi que ceux de M. Collignon et de E. Babelon, il finit par abandonner complètement les affaires et l'usine de fabrique d'alun, qu'il avait achetée en 1868 à sa sortie de l'École des Mines, pour se consacrer entièrement à son idée de constituer " un Musée de l'Hellénisme antique et modeme » à Paris Cette ambition muséographique, explique l'ampleur, la qualité, la richesse et la cohérence de cette collection systématique qui devait d'ailleurs être complétée par des moulages et des photographies. Il s'agissait, dans l'esprit de son inventeur, de montrer l'influence de l'art grec sur les civilisations qui lui ont succédé. Une fois acquis, les objets étaient restaurés, présentés et rangés dans des vitrines. Esprit original, auteur de poèmes inspirés par la Grèce antique («A une statue grecque », "A l'Hellas », "Au golfe de Corinthe », "A mon maître Edmond Pottier ») et d'articles défendant la cause de la Grèce contemporaine, il avait conçu pour son "Musée nouveau » un projet d'une grande modernité : dans des salles séparées par des cloisons mobiles et éclairées par des plafonds vitrés, il aurait voulu donner un aperçu géographique de la Grèce par la présentation de peintures ou de photographies de paysage, distinguer soigneusement les originaux grecs des copies «affadies » de l'époque romaine, présenter côte à côte les sculptures de marbre ou de bronze, les figurines en terre cuite, les vases, les monnaies et les entailles, disposer en regard des oeuvres originales des moulages, constituer une bibliothèque d'accès libre. Dès avant sa mort, en 1921, il songea à léguer sa collection à un grand musée parisien. Mais les conditions du legs testamentaire, et notamment l'impossibilité de disperser les objets qu'il avait rassemblés dans différentes collections déjà constituées, conduisirent le Louvre, puis le Musée Guimet, à refuser le legs. C'est finalement dans sa ville natale quaboutit la collection en 1937. Une partie de la collection de céramique a déjà été publiée : J. DE LA Genière, CVA, Laon, I ; A. J. DECAUdin, Les antiquités chypriotes dans les collections publiques françaises, Nicosie, 1987, p. 51-84; R. M. COOK, "A wild goat oinochoe in Laon ", EYMOYZIA, Ceramic and Iconographic Studies in Honour of Alexander Cambitoglou, édit. Jean-Paul Descoeudres, Sydney, 1990, p. 55-56. Un catalogue de la collection de sculptures est actuellement en préparation. Pour le projet de musée de l'Hellénisme antique et moderne, cf. P. Margulerite de la Charionie, "Création d'un Musée de l'Hellénisme antique et modeme, Nature, Arts, Littérature ", RA, 1915, 2, p. 228-235.

6. Le faîte du fronton n'est pas conservé, mais la composition du fragment conservé pernet de restituer approximativement à la stèle une largeur initiale d'environ $0,35 \mathrm{~m}$. 
Musée de Laon, numéro d'inventaire : 371546.

Marbre du Pentélique. Cassure verticale irrégulière à droite, légèrement à gauche de l'axe de la stèle ; cassure horizontale sous la vignette sculptée, au début du texte inscrit (on a sans doute scié la stèle pour vendre plus facilement le relief sans l'inscription : la partic avant du lit de pose est parfaitement lissće, la partic arrière taillée finement à la gradine, de manière à constituer une surface de pose régulière et horizontale). Le bord supéricur ainsi que le côté gauche, taillé à la gradine, sont intacts. La face postéricure est dégrossic à la pointe.

Hautcur maximale conservée : 0, 455 ; largeur maximale (mesurée au bandeau horizontal du fronton) : 0,172 ; largcur sous le fronton : 0,145 ; largeur à la base de la stèle : 0,165 ; hauteur conservée du fronton : 0,075 ; épaisscur maximale : 0,11 .

Champ sculpté : hautcur : 0,25 ; largeur conservéc : en haut 0,105 , en bas 0,11 ; profondeur : 0,002 .

L'état de conservation n'est pas excellent. L'acrotère latéral est endommagé. Les plis du péplos sont légèrement effacés sur la jambe droitc. La surface est par endroits recouverte d'une pellicule de concrétions.

\section{I. - LE RELIEF.}

La vignette sculptée était encadrée par deux pilastres couronnés de chapiteaux doriques et par un entablement surmonté d'un fronton orné d'acrotères et composé d'un tympan encadré par deux geisa taillés en bandeau en légère saillie. Un ovolo (hauteur : 0,025) séparait le fronton d'un bandeau plat (hauteur : 0,025) figurant l'épistyle. Ces éléments d'architecture à peine suggérés en très bas relief étaient sans doute rehaussés de peinture : l'ovolo avait peut-être reçu une frise peinte d'oves, comme sur une stèle à en-tête trouvée sur l'Agora ${ }^{7}$. De même, la hauteur inhabituelle de l'entablement pourrait s'expliquer par la représentation en peinture d'une architrave et d'une frise ${ }^{8}$.

A l'intérieur de ce cadre architectural, Athéna, que l'on reconnaît à l'égide esquissée sur sa poitrine, se tient debout, le corps de trois-quarts et la tête de profil, tournée vers la droite (Fig. 2). Le casque corinthien relevé, elle est appuyée sur la jambe gauche, la jambe droite étant à peine fléchie et déportée de côté. Elle est vêtue d'un péplos dont le long rabat, serré sous la poitrine par une ceinture qui dessine un demi-cintre, forme deux bouffants sur les flancs. Son bras droit levé s'appuie sur une lance qui était peinte. Elle retient de sa main gauche un bouclier posé à terre verticalement, représenté en biais et vu de l'intéricur.

La déesse ne présente aucun hanchement visible. C'est à peine si la position du bras gauche, dont on voit la partie interne, suggère une très légère rotation du torse et de l'épaule gauche en partic masquée. Cette attitude statique, la raideur des plis du péplos qui souligne l'axe du corps, la position de la lance, presque parallèle au corps d'Athéna, confèrent une certaine raideur à la

7. Agora I 6524. Cf. B. D. MeritT, Hesp. 21, 1952, p. 355-359, pl. 89-90; pour d'autres commentaires sur cette stèle, par ailleurs très proche de celle de Laon, cf. infra, p. 7 et fig. 6.

8. Cette architecture fictive et miniature, qui se développe à partir de la fin du Ve siècle sur les stèles funéraires et les reliefs votifs, où parfois les détails de l'ordre étaient rendus par la peinture (rouge, bleu et jaune), doit être ajoutée au dossier discuté par $\Lambda$. Rouveret sur la "naissance d'un décor illusionniste " et l'apparition des imitations d'architecture en trompe l'oeil : Histoire et imaginaire de la peinture ancienne, Rome, 1989, p. 171. 
composition. Pourtant, quelques détails animent un peu l'ensemble. En effet, à l'intéricur d'une disposition où la verticalité domine largement, les plis du péplos sur la jambe gauche forment un axe très légèrement ouvert sur la droite qui s'oppose à celui donné par la lance, que le geste d'Athéna tient plus écartée sous le fer qu'à la base. L'ensemble du relief donne cependant une impression de rigidité.

Cette vignette sans originalité participe d'une production artisanale stéréotypée, ce qui explique certaines maladresses dans la mise en place d'Athéna à l'intéricur du champ sculpté. C'est ainsi que le casque corinthien relevé de la déesse déborde sur la limite supérieure de la vignette. Surtout, la position de la main droite d'Athéna est trop haute, compte tenu de la manière dont elle tient sa lance normalement, c'est-à-dire assez haut sur la hampe, mais avant le fer. En effet, la main touche ici la limite supéricure du champ sculpté, et il n'y a plus d'espace pour représenter entre l'une et l'autre le haut de la hampe et le fer. L'extrémité de la lance devait être peinte sur l'épistyle. Ce relief n'est pourtant pas sans qualité. La pondération d'Athéna représentée très légèrement de trois quarts, le mouvement de sa tête, le bouclier incliné et posé en biais, créent une certaine profondeur qui anime la composition en figurant une série de plans et en suggérant une ligne de fuite au centre du relief. La bonne qualité de ce très bas relief se remarque par ailleurs au modelé fin du bras et du visage.

Cette vignette, où une Athéna à peine modelée ne fait pas saillie par rapport à un encadrement architectural tout juste figuré par le relief et souligné par la pcinture, est sculptée comme un cartouche. C'était également la polychromic qui permettait de détacher la déesse du fond et de distinguer ses attributs, comme elle permettait de préciser les différents éléments du cadre architectural9.

L'interprétation de ce relief ne pose pas de problème. La représentation d'Athéna en armes correspond à l'iconographie classique des vignettes athéniennes d'en-tête de décret ${ }^{10}$ et les deux premières lignes d'une inscription sous le champ sculpté confirment, s'il en était besoin, cette interprétation.

Athéna, personnification d'Athènes et de ses institutions politiques, est la figure de très lọin la plus représentée sur ce type de stèles. Elle est connue dès l'époque archaïque avec de „ombreuses épiclèses civiques, Polias, Archégétis, Phratria, Boulaia. Son image fut utilisée systématiquement par la cité d'Athènes après les Guerres médiques. Son iconographie, qui sppartenait désormais presque uniquement à l'imagerie politique, se constitua autour des grandes consécrations commandées par la cité d'Athènes à Phidias qui servaient désormais de prototypes : Athéna Promachos, Athéna Parthénos, Athéna Lemnia ${ }^{11}$. Athéna était, comme à lépoque archaïque, toujours arméc, mais non plus gucrrière. C'était désormais une déesse paisible dont la victoire était déjà assurée et dont les armes n'étaient plus brandies en direction de 'ennemi, mais posées à terre. Ce sont ces types iconographiques qui furent utilisés pour les fécrets à relief avec de très faibles variantes ${ }^{12}$. La représentation d'une action ou la présence d'un

9. De même que sur un relief conservé à l'EF^ (inv. I-6) où B. Holtzmann avait décrit des traces de peinture bleue et puge, of. "Collection de l'École française d'Athènes », BCH 96, 1972, p. 73.

10. B. Hol.tzmann, "Collection de l'ícole Française d'Athènes: sculptures », BCH 96, 1972, p. 74 et n. ${ }^{\circ} 1$. La rovenance d'Athènes est assurée par la présence d'Athéna. Sur les deux cent vingt six en-tête de décret à relief connus à ce pur, Athéna n'est représentée que sur un seul parmi les dix-neuf qui viennent d'une autre cité. Encore s'agit-il d'un relief ilustrant une proxénic accordée par la cité de Delphes en l'honneur d'un citoyen athénien (cf. M. -A. ZAGDOUN, FD, IV h. Paris, 1977, p. 49-57 el fig. 32).

11. P. Dimargini, LIMC, II 1, 1984, s. v. Athéna, p. 1037-1038.

12. Mais également dans les reliefs votifs, dont l'iconographie n'est pas très différente. 
autre personnage ont parfois obligé le sculpteur à remanier et à adapter légèrement le modèle original en ajoutant un geste (dexiôsis ou couronnement).

Pourtant, malgré une iconographie simple et organisée en séries répétitives, l'Athéna du relief de Laon est difficile à classer dans la typologie définie par M. Meyer ${ }^{13}$. Elle n'appartient sûrement pas aux types de l'Athéna appuyée sur son bouclier, de l'Athéna assise ni à celui de l'Athéna portant son bouclier. Restent deux catégories entre lesquelles il est difficile de choisir : l'Athéna au bouclier et l'Athéna à la lance. Participant de ces deux types puisqu'elle s'appuie sur sa lance tout en retenant son bouclier posé à terre, elle présente une confusion entre deux séries iconographiques qui semblent bien, dans ce type de reliefs, être le plus souvent exclusives l'une de l'autre : quand la déesse porte la lance, clle ne porte pas le bouclier et vice-versa. M. Meyer a cru pouvoir mettre en relation chacune de ces catégories de reliefs avec un prototype : l'Athéna Parthénos serait celui des Athéna retenant leur bouclier posé à terre et l'Athéna Promachos celui des Athéna à la lance. Pourtant ces types sont, à mon sens, moins rigides qu'elle ne le pense : à condition de respecter un cadre général, constitué de références acceptables par la cité et lisibles par tous, et fixé, moins par une filiation stricte avec les grandes consécrations de l'Acropole, que par une culture iconographique qui permettait à tout Athénien de reconnaître, même avec une attention distraite, dans une péplophore armée ou casquée l'Athéna Polias, l'artisan jouissait d'une certaine liberté. Ainsi les variations sur le thème de l'Athéna civique ont pu jouer de la présence alternéc ou simultanéc de la lance et du bouclier et de leurs positions respectives. Quelques œuvres appartenant à la grande statuaire présentent d'ailleurs, comme le relief du musée de Laon, des caractéristiques communes au type de la Parthénos, avec son bouclier posé à terre, et à celui de la Promachos, avec sa lance. Il s'agit de l'Athéna Médicis, appelée aussi Minerve Ingres ${ }^{14}$, dont l'original est attribué à Phidias ou à son cercle, et d'une statue d'AthénaAllât, trouvée à Palmyre ${ }^{15}$. Comme pour les Marianne de la IIIe République ${ }^{16}$, il n'y avait pas un type officiel à proprement parler, mais plutôt un genre iconographique, celui de l'allégorie civique. N'oublions pas que ces images n'étaient pas destinćes à représenter officiellement la Cité à l'étranger ${ }^{17}$. Les commanditaires, les réalisateurs et les destinataires, étaient des citoyens, sinon des habitants d'Athènes.

Le relief du musée de Laon ne trouve dans la catégorie des en-tête de décret à relief que deux parallèles iconographiques très proches : une vignette illustrant une inscription éphébique d'Athènes datant de 334/33318 (Fig. 3) et un relief surmontant un décret de la cité de Delphes

13. La synthèse de M. Meyer s'inscrit, d'une certaine manière, dans la tradition qui avec R. BiNNEBoESSEL (Studien zu den attischen Urkundenreliefs des 5. und 4. Jahrhunderts, Magdeburg, 1932) et H. K. SÜSSEROTT (Griechische Plastik des 4. Jahrhunderts v. Chr., Francfort, 1938) cherchait surtout établir à partir d'oeuvres sculptées et peintes bien datées la chronologie absolue de l'art grec au IVe siècle.

14. F. ChamouX, "Le type de la Minerve Ingres », BCH 68-69, 1944-1945, p. 206-239.

15. A. J. N. PRAG, "New Copies of the Athena Parthenos from the Fast », Parthenon Kongress Basel, Mayence, 1984, p. $182-187$ et pl. $10-11$.

16. M. Agulhon, Marianne au combat, l'imagerie el la symbolique républicaines de 1789 à 1880 , Paris, 1979 et Marianne au pouvoir, l'imagerie et la symbolique républicaines de 1880 à 1914, Paris, 1989.

17. Ou la République. Si l'on veut trouver une représentation civique officielle, c'est plutôt sur les types monétaires, sur les sceaux qui étaient apposés sur les décrets, sur les parasèmes de certains décrets honorifiques, parfois sur les boucliers qu'il faudrait les rechercher. Il faut à mon sens distinguer strictement l'emblème précis de la cité et le répertoire civique, plus large et plus étendu. Sur les emblèmes officiels, ef : L. LACROIX, "Les blasons dans les villes grecques", Etudes d'Archéologie Classique I, 1955-1956, p. 91-115; T. RITTI, "Sigle ed emblemi sui decreti onorari greci ", Atti dell'Accademia Nazionale dei Lincei, Memorie, serie VIII, vol. XIV, Rome, 1969, p. 259-360.

18. Mus. Fipigr. Ath. inv. 2802a ; M. MFYlR, op. cit., A 99, p. $293-294$ et pl. 31, 1 ; pour l'inscription, cf. IG, $\mathrm{II}^{2}, 2970$ 
accordant la proxénie à un citoyen athénien, daté autour de $330^{19}$ (Fig. 4). Dans les trois cas, une Athéna de face appuic son bras droit levé sur sa lance et retient son bouclier, posé à terre de trois quarts et vu de l'intéricur. La pondération de la déesse, sa place à gauche de la vignette sculptée et l'absence de geste en direction du personnage représenté dans la moitié droite, aujourd'hui disparu dans les reliefs de Laon et d'Athènes, se retrouvent sur les trois stèles. La position du bouclier qu'Athéna retient de la main gauche, représenté de trois quarts et en partie caché par le corps de la déesse est également la même dans les trois monuments ${ }^{20}$. La scule différence réside dans l'encadrement architectural : sur les stèles de Delphes et d'Athènes, le champ sculpté est limité en bas par une moulure en saillie par rapport au reste de la stèle inscrite, alors que dans le relief de Laon, la vignette ne présente aucune moulure en relief.

La première approximation chronologique que l'on peut tirer de ces rapprochements iconographiques est confirmée par les analogies stylistiques que présente le relief du musée de Laon avec trois autres en-tête de décret à relief datés du troisième quart du IVe siècle. Deux de ces reliefs sont datés précisément par les inscriptions qu'ils couronnent : un relief conservé au musée d'Avignon ${ }^{21}$ daté de 339/338 (Fig. 5) et une stèle découverte sur l'Agora d'Athènes datée de 337/33622 (Fig. 6). Le troisième, conservé à l'École française d'Athènes, est daté par un rapprochement stylistique avec les deux reliefs précédents ${ }^{23}$ (Fig. 7). Sur ces trois documents, on retrouve les mêmes proportions et les mêmes éléments d'encadrement : profil figurant un chapiteau de pilastre, ovolo, fronton avec tympan en creux surmonté par des acrotères. La ressemblance de l'Athéna d'Avignon avec celle de Laon - même pondération, dessin identique du costume, même style graphique - renforce d'autre part l'hypothèse d'une proximité chronologique entre ces reliefs. Cette parenté de style et de composition ${ }^{24}$ permet de proposer pour le relief de Laon une date vers 340/330 et de formuler l'hypothèse qu'ils sortent du même atelier ${ }^{25}$.

Parfois, l'iconographie de la vignette sculptée était en rapport avec le contenu du décret qu'elle surmontait. Les traités d'alliance étaient souvent illustrés par la représentation d'une poignée de main entre Athéna et la divinité poliade de la cité avec laquelle Athènes concluait une alliance, les proxénies ou les décrets honorifiques par celle de la déesse couronnant un personnage ${ }^{26}$. Le relief de Laon n'entrant dans aucune de ces catégories, il est difficile de p. 300

19. Mus. Delphes inv. 2707 ; M.-A. ZAgdoun, FD, IV 6, 1977, n ${ }^{\circ} 14$ p. 49-57; M. MEYER, op. cit., A 123,

20. M. MEYer, op. cit., p. 166 n. 1137 a classé les vignettes sculptées des stèles d'Athènes et de Delphes dans la catégorie des Athéna au bouclier. Elle pense, p. 169, que la présence d'une lance relève de la libené de l'artisan avec le y pe qui lui était imposé. Il me semble qu'il est plus satisfaisant d'expliquer la relative variété des Athéna des en-tête de Jécret par le caractère peu contraignant du répertoire de l'allégorie civique. La typologie iconographique constituée par es savants modemes était à mon sens étrangère aux utilisateurs antiques.

21. Musée d'Avignon, inv. 38. Cf. Th. Reinach, REG 13, 1900, p. 158-169 ct pl. II ; R. Binneboessel, Studien zu len attischen Urkundenreliefs des 5. und 4. Jahrhunderts v. Chr, Leipzig, 1932, p. $13 \mathrm{n}^{\circ} 55$ (pour le commentaire, if. p. 63-65) ; H. K. SüsSEROTT, Griechische Plastik des 4. Jahrhunderts v. Chr., Francfort, 1938, p. 85 et pl. 5-1; M. GuarducCi, Epigrafia Greca, II, Rome, 1969, p. 595 et fig. 187 ; M. MEY:R, Die griechischen Urkundenreliefs, Berlin, A 91 p. 291 ; pour l'inscription, cf. $I G \mathrm{II}^{2}, 231$.

22. Agora I 65254. Cf. B. D. MERITT, Hesp. 21, 1952, p. 355-359, pl. 89-90 ; L. HaussmanN, Griechische Weihreliefs, Berlin, 1960, p. 44 et fig. 22 (une photographie du relief a été publiée dans Hesp. 22, 1953, pl. 20); M. MEYER, op. cit., A 97 p. 293 et pl. 30, 2.

23. Collection de l'École française d'Athènes, inv. I-6. Cf. B. HoltzmanN, "Collection de l'École française f'Athènes : sculpture ", BCH 96, 1972, p. 73-79, fig. 1 et 2 ; M. MFYER, op. cit., A 137 p. 304 et pl. $41,2$.

24. Ces reliefs ont déjà été rapprochés par J. Frel, Les sculpteurs attiques anonymes, 430-300, Prague, 1969 ; G. Kalogeropoulou et E. Vanderpool, Arch. Delt. 25 (1970), p. 204-216 et pl. 70-71 ; B. Hol.tzamann, op. cit.

25. Cf. B. HOlTZMANN, op. cit.

26. I. KASPER-BUTZ, op. cit., p. 59-132. 
restituer la nature et l'attitude du personnage qui faisait face à la déesse dans la partie droite de la vignette. La position d'Athéna debout, l'absence de geste en direction de son interlocuteur, son regard droit permettent seulement d'imaginer un personnage debout regardant vers la gauche. S'agissait-il d'un citoyen étranger honoré par une proxénie, d'une allégorie civique ou d'une autre divinité ? Seuls l'analyse et le commentaire de l'inscription pourraient fournir les éléments d'une réponse.

\section{II - L'INSCRIPTION.}

L'inscription nous est parvenue dans un état si fragmentaire qu'il n'est pas permis de faire la moindre hypothèse sur le contenu exact du décret. N'est en effet conservé que le début de ses deux premières lignes et les lettres très effacées n'autorisent une lecture assurée que pour la première :

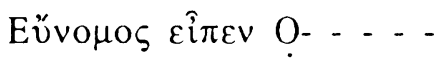

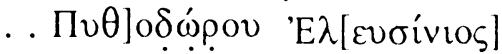

Notes critiques : L. 1 : la lecture de o n'est pas sûre et n'est confirmée par aucune restitution satisfaisante : ö[ $\pi \omega \varsigma]$ ? L. 2 : la lecture des lettres pointées, qui ne sont pas complètes sur la pierre, me semble assurće.

Ce texte présente à première vue deux particularités : le décret commence par le nom du rogator et la formule de proposition; le nom du rogator n'est suivi ni de son patronyme ni de son démotique. Il s'agit donc d'une copie très abrégée de l'intitulé officiel d'un décret attique.

A partir du IVe siècle, les décrets inscrits complets comprenaient un titre, une formule d'invocation, un intitulé (le nom de l'archonte, la tribu exerçant la prytanie, le nom de son secrétaire, la date de la prytanie, la qualité de l'assemblée, le nom du président de séance), une formule de sanction, le nom de l'auteur de la proposition suivi de la formule de proposition, les considérants, la formule hortative et enfin le dispositif27. Parfois, une partie du formulaire était abrégée et manquait alors tel ou tel ćlément de l'intitulé28. Ces variations que l'on constate sur les décrets inscrits ne tenaient pas à l'absence de formalisme juridique des Grecs, qui se seraient accommodés d'une diplomatique sans rigueur, mais plutôt à la nature des documents que nous lisons. Le texte inscrit sur la pierre n'était qu'une copie du texte voté et déposé aux archives. C'était le texte des archives qui ćtait le document officiel, le texte normatif qui avait une valeur juridique et dont la diplomatique devait assurer l'authenticité et la légalité. C'était lui qui devait être enregistré et archivé pour pouvoir y être éventucllement consulté, à Athènes généralement dans le Métrôon ${ }^{29}$ mais aussi dans d'autres bâtiments ${ }^{30}$ où il était classé selon la séance, la prytanie et l'archontat. La publicité par voie d'affichage donnée aux textes votés était loin d'être

27. Sur le formulaire des décrets attiques, cf. F. X. DRUE:, "Le préambule des décrets attiques », Revue Belge de Philologie et d'Histoire 49, 1971, p. 73-83 et le compte rendu de J. et L. ROBERT, Bull. Epigr., 1976, 173 ; P. J. RHODES, The Athenian Boule, 1972, p. 64-71 et 244-246; J. BINGEN, "Préambule et promoteur dans le décret attique ", Hommages à C. PRÉAUX, Bruxelles, 1975, p. 470-479; A. S. HeNky, The Prescripts of Athenian Decrees, Leyde, 1977.

28. Voir les exemples de formulaires abrégés rassemblés par A. S. HENRY, op. cit., p. 32

29. H. A. ThOMPSON et R. E. WyChERLi:y, Agora XIV, 1972, p. 35-36 ; J. M. CA.MP, The Athenian Agora, Londres, 1986, p. $91-94$.

30. S. GeORGOUDI, "Manières d'archivage et archives de cités ", Les savoirs de l'écriture en Grèce ancienne, édit. M. Détienne, Lille, 1988, p. 221-247, en particulicr, p. 227-233 
automatique. Dans une démocratie directe où tous les citoyens, en théorie au moins, participaient régulièrement aux assemblées, il n'était pas indispensable de faire connaître des décrets qui avaient été votés au vu et au su du peuple assemblé. La publicité des règles de droit, indispensable non seulement à la démocratie mais aussi à la stabilité et à la bonne marche des rapports juridiques entre les citoyens, était assurée par la présence des citoyens à toutes les assemblées. Les inscriptions s'adressaient à l'opinion publique, c'est-à-dire aux gouvernés, mais celle-ci comprenait les gouvernants cux-mêmes. On peut d'ailleurs penser qu'un citoyen absentéiste se plaignant d'ignorer tel ou tel dispositif juridique aurait pu se voir reprocher d'avoir manqué à ses obligations civiques et l'adage : «nul n'est censé ignorer la loi » se comprend aisément dans le cadre d'une démocratic gouvernante.

Une fois prise la décision de publier un décret, l'inscription sur la pierre, qui lui assurait une pérennité et une valeur importante, n'était pas la seule voie possible. Le texte pouvait n'être que

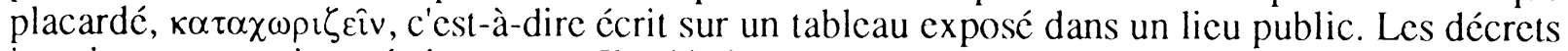
inscrits sur une pierre étaient rares. Ils n'étaient pas un journal mural. Ils n'avaient pas comme mandat spécifique d'instruire un public, mais de satisfaire un désir de renommée chez des lecteurs immédiats en recourant à un support durable, accessible et public. N'étant pas destinées à être consultées mais à être vues et lues ${ }^{31}$, les inscriptions n'étaient pas la transcription fidèle du texte voté, mais souvent celle d'un texte abrégé que l'on devait seulement pouvoir reconnaître et comprendre. Elles n'étaient qu'une " copie sur pierre », une " ombre de décret dans la caverne des épigraphistes ${ }^{32}$. Seules quelques indications restaient indispensables à la bonne intelligence du décret quand il ćtait inscrit sur pierre. Il s'agissait surtout du nom du rogator qui permettait de reconnaître facilement le texte en question et qui lui assurait d'ailleurs sa publicité. Dans les débats d'un procès ou d'une assemblée, les décrets étaient en effet désignés normalement par le nom de l'autcur de la proposition ${ }^{33}$. C'est le cas dans la plupart des régimes d'assemblée où le débat public autour d'un texte tend à le personnaliser en opposant à son auteur, qui doit le défendre, la masse des élus, qui doivent l'approuver ou le refuser par un vote. C'est ainsi que sous la Convention, on parlait des décrets Barrère et qu'aujourd'hui encore on parle de la loi Marthe Richard. La syntaxe même des textes votés par les assemblées grecques contribuait à donner au promoteur d'un texte une place de premier plan puisque le contenu du décret

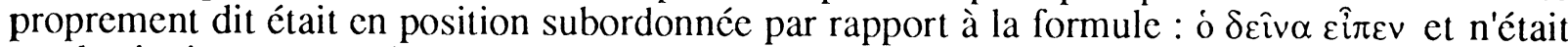
que la citation transposéc au style indirect du contenu exact de la proposition. Enfin, l'orateur qui avait proposé un projet de décret était responsable en cas d'exception d'illégalité. Il était poursuivi en cas de $\gamma \rho \alpha \varphi \grave{\eta} \pi \alpha \rho \alpha v o ́ \mu \omega v$. Une fois un texte adopté par l'assemblée, l'orateur pouvait éventuellement bénéficier d'un vote de confiance, sous la forme d'un décret honorifique, mais tout aussi bien être l'objet d'un vote de défiance : l'exception d'illégalité, procédure fréquente que tous les hommes politiques du IVe siècle ont dû affronter, souvent plusieurs fois pendant leur carrière, constituait le pendant négatif du décret honorifique ${ }^{34}$. La prééminence de

31. Encore que certaines inscriptions, en particulier celles qui étaient gravées sur des murs, ne pouvaient être lues par un simple passant. Ainsi, les décrets de proxénie ou les décrets amphictioniques inscrits sur les demières assises du mur polygonal, à Delphes, sont illisibles du pied du mur. Aussi faut-il faire une différence entre les stèles inscrites, qui exposaient un texte devant un public et remplissaient ainsi un rôle d'ostentation, et certaines inscriptions, gravées de telle manière qu'elles étaient inaccessibles, dont la fonction étaient de consacrer ou de pérenniser un texte en l'associant à un sanctuaire ou à un monument important. On rapporte que Georges Daux disait des inscriptions qu'elles n'étaient pas faites pour être lues mais pour être là.

32. J. Bingen, loc cit.

33. Par exemple, Andocide, Sur les Mystères, 77-79 ou 83-84

34. C'est ainsi, par exemple, que dans son plaidoyer contre Ctésiphon Eschine cherche à transformer un décret honorifique en l'honneur de Démosthène en vote de condamnation de sa politique (cf. P. Carlit: Démosthène, Paris, 1990, p. 231). 
l'orateur-auteur de la proposition ${ }^{35}$, qui est l'homme politique par excellence à Athènes, dans le formulaire des stèles inscrites s'explique donc aisément.

Rien d'étonnant donc à trouver sur la stèle à en-tête de décret du musée de Laon une inscription commençant par le nom de l'auteur de la proposition. Reste que parmi tous les degrés d'abréviation possible d'un décret, ce préambule présente une forme presque minimale. Or les intitulés très résumés ou très simplifiés se trouvent émaner souvent de subdivisions civiques comme le dème ou la tribu. Ainsi dans les décrets de tribus, les intitulés abrégés étaient fréquents. On y trouve la formule de sanction ou le nom du proposant, parfois les deux, parfois aussi l'invocation aux dicux. L'ordre de l'intitulé n'y était pas non plus très rigoureusement fixé.

Dans tous ces décrets émanant de subdivisions civiques, mais aussi dans ccux votés par des associations privées ${ }^{36}$, il n'est pas rare de trouver des noms abrégés, c'est-à-dire un nom suivi d'un démotique abrégé ou même un nom scul sans patronyme ni démotique. Ainsi, dans les décrets de tribu, le nom du rogator était parfois scul ${ }^{37}$, de même dans les décrets de dèmes ${ }^{38}$, les décrets de clérouchies ${ }^{39}$, les décrets de famille ${ }^{40}$, les décrets de phratries ${ }^{41}$ ou les décrets d'associations religicuses ${ }^{42}$. On pourrait croire que la présence d'un relief illustrant la décision donnait une solennité supplémentaire au texte inscrit et que le décret transcrit sur une stèle à entête était plus complet que sur une stèle ordinaire. Mais là encore, le catalogue établi par M. Meyer permet de retrouver facilement de nombreux parallèles à l'intitulé minimal du texte gravé sous le relief du musée de Laon.

En effet, sur un certain nombre d'exemples, le texte inscrit commencait directement soit par le nom du secrétaire ${ }^{43}$, soit par le nom de l'auteur de la proposition de décret ${ }^{44}$, avec des formes d'ailleurs plus ou moins complètes (nom seul, nom avec patronyme ou démotique, parfois les deux). Un décret surmonté d'un relief du dème d'Eleusis voté en 319/318 en l'honneur du

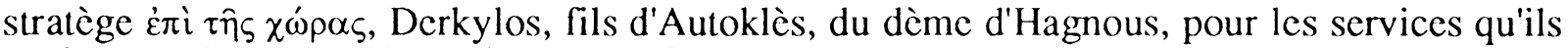
avaient rendus dans l'éducation des enfants du dème, est de ce point de vue très proche de

35. M. H. Hansen, The Sovereignty of the People's Court in Athens in the Fourth Century B. C. and the Public Action against Unconstitutional Proposals, Odense, 1974, p. 22-23.

36. Les décrets des associations privées, qui sont le plus souvent des associations de cultes, sont rédigés sur le modèle des décrets publics.

37. Par exemple, $I G \mathrm{II}^{2}, 1138$ (403/402), 1139 (403/402), 1145 (353/352 ?), 1146 (1 $1^{\text {ère }}$ moitié du IVe siècle), 1147 (1 $^{\text {ère }}$ moitié du IVe siècle), 1148 (1 ${ }^{\text {ere }}$ moitié du IVe siècle), 1149 (1 ${ }^{\text {ère }}$ moitié du IV ${ }^{\mathrm{e}}$ siècle), 1151 (milieu du IVe siècle), $1156(334 / 333)$ etc.

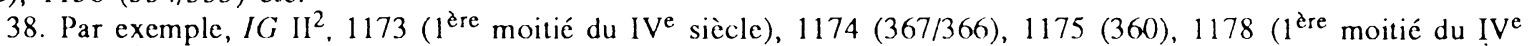

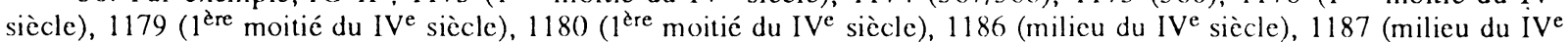
siècle), 1190 ( $2^{\text {ère }}$ moitié du IV siècle) etc.

39. Par exemple, $I G \mathrm{II}^{2}, 1225$ (250 ca.).

40. Par exemple, $I G \mathrm{II}^{2}, 1231$ (fin du IV $\mathrm{V}^{\mathrm{e}}$ siècle).

41. Par exemple, $I G \mathrm{II}^{2}, 1241(300 / 299)$.

42. Par exemple, $I G \mathrm{II}^{2}, 1256(329 / 328)$

43. Autour de 430: M. MiYlir, op. cit., A 28 et pl. 9, 1 =IG, II 2188 ; en 410 : M. MFYYer, op. cit., A 39 et pl. 9 , $2,=$ M. B. Walbank, Athenian Proxenies of the 5 th Century BC, 1978, p. 487 et R. S. Stroud, Hesp. 48, 1979, p. 180193 ; en 406/405: M. MtYer, op. cit., A 22 et pl. 8, $1=I G, I^{2}, 124$; au IVe siècle : M. MEYER, op. cit., A $166=I G$ II 2 90 ; en 378/377 : M. MEYER, op. cit., A $48=I G$ II ${ }^{2}, 277$.

44. Autour de 330: M. MeYl:R, A 117 et pl. 38, $1=I G \mathrm{II}^{2}, 1193$; dans le $3^{\text {ème }}$ quart du IVe siècle : M. MEYER,

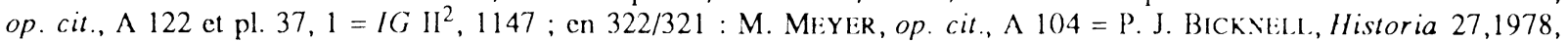
p. 369-374 ; en 313/312: M. MEYER, op. cit., A 141 et pl. 44, 2 = W. PEEK et N. KYPARISSIS, "Attische Unkunden, AM 66,1941, p. $218-219$ et pl. 73 ; à la fin du IVe siècle, un décret honorifique émanant sans doute d'une association religieuse si l'on en juge par l'iconographie du relief qui représente deux fidèles dans le geste de l'adoration : M. MEYER, op. cit., A $156=I G$ II 2, 599 ; au début du III ${ }^{\mathrm{e}}$ siècle : M. MEYER, op. cit., N $17=I G$ XII, 9, 213. 
l'inscription de Laon $^{45}$. L'initiative du vote vint d'un parent d'élève, Philippos. On y trouve la même formule de proposition que sur l'inscription du relief de Laon : le nom du rogator n'est pas suivi de son patronyme ou de son démotique. Cette place de premier plan donnée au secrétaire jusque dans le premier tiers du IVe siècle puis au rogator ${ }^{46}$ confirme, à mon sens, l'idée de M. Meyer sclon laquelle ces en-tête à relief seraient dûs à la libéralité du secrétaire de séance dans un premier temps, puis de l'autcur de la proposition. L'absence de clauses financières spécifiques indique que le financement d'un tel monument relevait de l'initiative privée et non des décisions votées par l'assembléc : de fait, aucune des inscriptions surmontées d'un relief ne contient de clause particulière concernant la facture de celui-ci ${ }^{47}$. Le prix de la gravure ne changeait pas selon que la stèle était ou non surmontée d'une vignette, en sorte que si le relief n'est pas conservé, rien ne permet plus aujourd'hui de supposer qu'il s'agit d'un décret à en-tête. Sur le décret du dème d'Elcusis en l'honneur de Derkylos, le texte comprend, comme il est habituel, la décision d'accorder les honneurs et parmi cux l'ordre de graver le décret, dont l'exécution est confiće aux pères des ćlèves. On ne lit aucune clause financière. Dans ce cas, où les protagonistes sont clairement désignés, on peut facilement reconstituer la procédure. Une fois le texte voté et la somme nécessaire à sa gravure affectéc, ce furent les parents d'élèves, collectivement, ou le rogator, personnellement, qui décidèrent de faire surmonter la stèle inscrite d'un relief. C'était donc une fois que le trésorier cut versé la somme de la fabrication et de la gravure de la stèle qu'intervenait, à titre individuel, le secrétaire de l'assemblée ou le rogator pour compléter par une libéralité personnelle l'argent public et faire figurer un en-tête au-dessus du décret. C'était donc bien un document public, puisque l'inscription était une décision politique votée par l'assemblée, mais un document public que l'initiative privée venait couronner.

La double particularité du début de l'intitulé de l'inscription du musée de Laon n'est donc pas une anomalic. Elle s'explique si l'on rapproche ce document notamment des inscriptions de décrets votés par les subdivisions civiques comme le dème ou la tribu par exemple et dont les intitulés sont très souvent abrégés.

L'iconographic du relief de Laon ne concorde pourtant pas parfaitement avec l'impression générale donnée par le corpus des décrets à relief votés par les tribus ou les dèmes. En effet les vignettes illustrant ce type de décrets, qui sont presque toujours des décrets honorifiques, montrent rarement Athéna. Le plus souvent, on y voit la divinité du dème (Démeter à Eleusis par excmple) ou son allćgoric ou le héros de la tribu en présence des personnages honorés.

45. IG II $\mathrm{II}^{2}, 1187$; F. W. Mitchel, Hesp. 33(1964), p. 337-351 ; J. et L. RoBerT, Bull. épigr., 1965, 136 ; M. Meyer, op. cit., A 133 p. $302-303$ et pl. $38,2$.

46. Parfois même, le nom du secrétaire est répété : il est signalé normalement dans le corps du décret mais aussi en fttres plus importantes au-dessus du relief ou en titre juste au-dessus de l'inscription où il n'a donc aucune valeur chronologique mais plutôt signalétique. Ainsi en est-il, dans le décret accordant vers 420 la proxénie à Proxénidès de (nide où le nom du secrétaire est inscrit 1. 2, juste sous le titre mais sans doute aussi dans le corps du décret : M. MEYER, pp. cit., A 6 et pl. $2=I G \mathrm{I}^{3}, 91$; dans le décret autorisant l'exercice du culte de la déesse thrace Bendis au Pirée entre 420 d 410 : M. MEYER, op. cit., A $12=I G \mathrm{I}^{3} 136$; dans le décret accordant la proxénie à des citoyens d'Abydos autour de $410:$ M. Meyer, op. cit. , A $18=I G \mathrm{II}^{2}, 49$; dans le décret voté pour les Samiens en $403:$ M. MEYER, op. cit., A 26 et p. $10,1=I G \mathrm{I}^{3}, 127$; dans le décret de proxénie voté en l'honneur de Simon de Béotie en 403/402: M. MEYER, op. cit., I $25=I G \mathrm{II}^{2}, 2$; dans le traité d'alliance entre Athènes et Argos, où le nom du secrétaire qui commence le décret est sans oute répété dans le texte du décret proprement dit: M. MEYER, op. cit., ^ 8 et pl. $3=I G$ I 3,86 .

47. Alors que la gravure d'une inscription sur une stèle appartient aux décisions politiques votées par l'assemblée (la formule óv $\alpha \gamma_{\rho} \dot{\alpha} \psi \alpha 1$. . est un alinéa du texte voté) et que le financement de la gravure, prévu dans le corps du texte, est public. 
La prosopographie ne permet pas de déterminer la nature de l'assemblée qui a voté le décret de Laon ni le contenu de ce dernier. Un Eunomos, fils d'Euthynomos, du dème de Gargettos, a été bouleute en $343^{48}$; un Eunomos, fils d'Euonymon, a été bouleute et hiérope en $335^{49}$. L'écart chronologique entre ces deux homonymes n'est pas tel que la datation stylistique du relief puisse permettre de pencher pour l'un plutôt que pour l'autre. Quant au patronyme que l'on peut restituer à la ligne 2, Pythodôros, qui est sans doute celui du personnage honoré, il est encore moins éclairant : rien ne permet de choisir entre les différents candidats déjà connus par ailleurs, sauf peut-être son démotique, largement restitué il est vrai. Un Pythodôros, fils d'Euphron, démote d'Eleusis ${ }^{50}$, est attesté comme naope à Delphes en $324 / 3^{51}$.

Quoique sans parallèle absolument exact, la stèle du musée de Laon s'intègre pourtant très bien dans la famille des en-tête de décrets à relief, un type de monuments formellement à michemin entre l'inscription publique et le relief votif privé. Les commanditaires, sinon les artisans, jouissaient d'une certaine liberté vis-à-vis de l'iconographie et du contenu de l'inscription. Les contraintes quant au contenu de l'inscription et à l'iconographie de la vignette sculptée, étaient licées au " public lecteur ». Ces monuments n'étaient pas des documents figurant une représentation officiclle de la divinité civique et reproduisant fidèlement un texte officiel, mais dus à une initiative politique individuclle, ils étaient destinés à donner à un décret, et surtout à son promoteur, une publicité doublement solennisće par l'inscription sur une stèle et la gravure d'un relief.

48. J. Kirchner, Prosopographia Altica n 5868, Berlin, 1903 ; R. Develin, Athenian Officials, 684-321 B. C., $1989, \mathrm{n}^{\circ} 1181$.

49. J. KiRCHNER, ibid., $n^{\circ} 5869$; R. DEVEl.IN, ibid., $n^{\circ} 1180$.

50. Id., ibid., ${ }^{\circ} 6125$.

51. J. Bousquet, Les comptes de Delphes, CID II, 1989, nº 102 II B 1.26. 


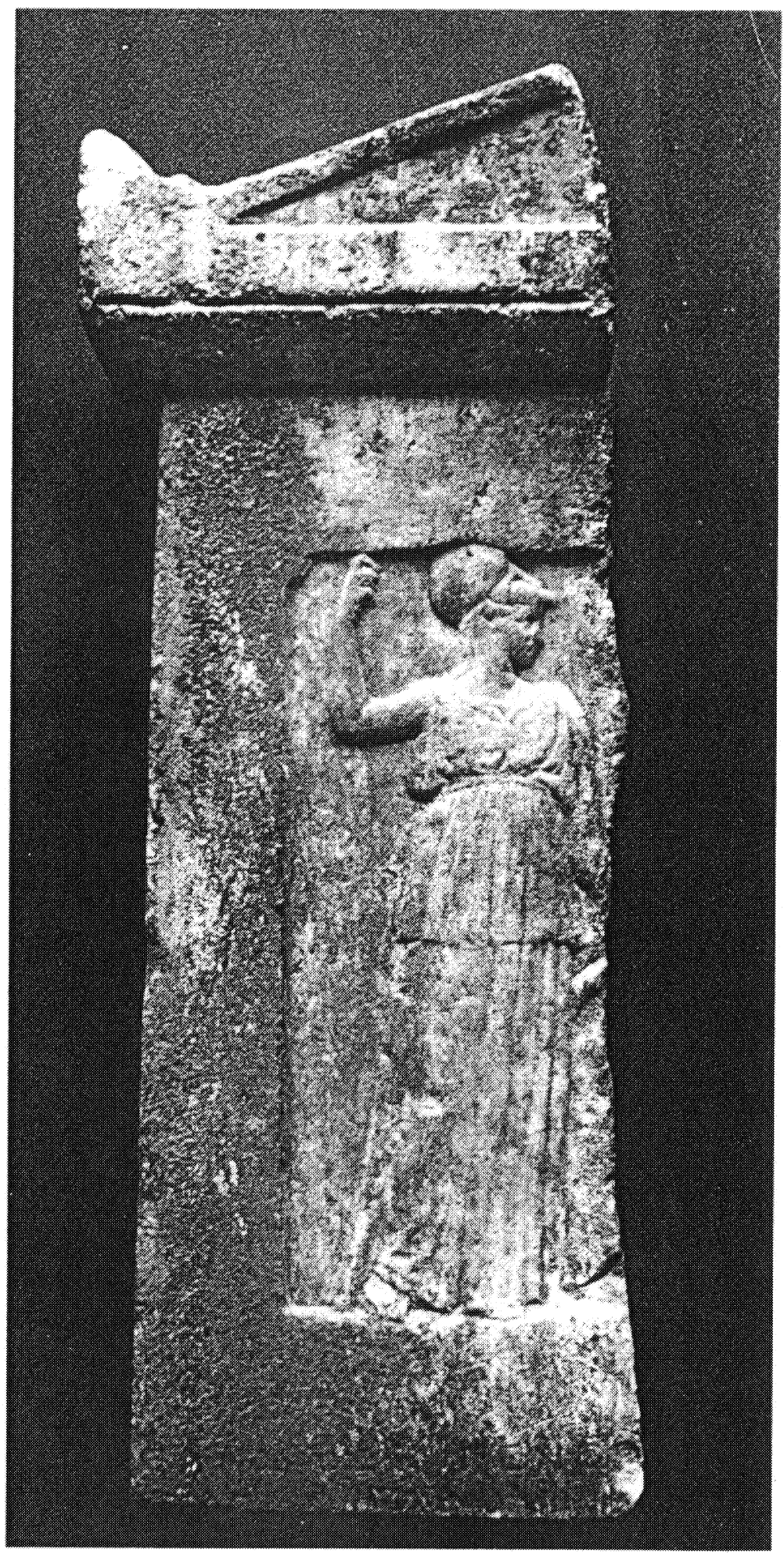

Fig. 1. - En-tête de décret, Musée de Lanon. 


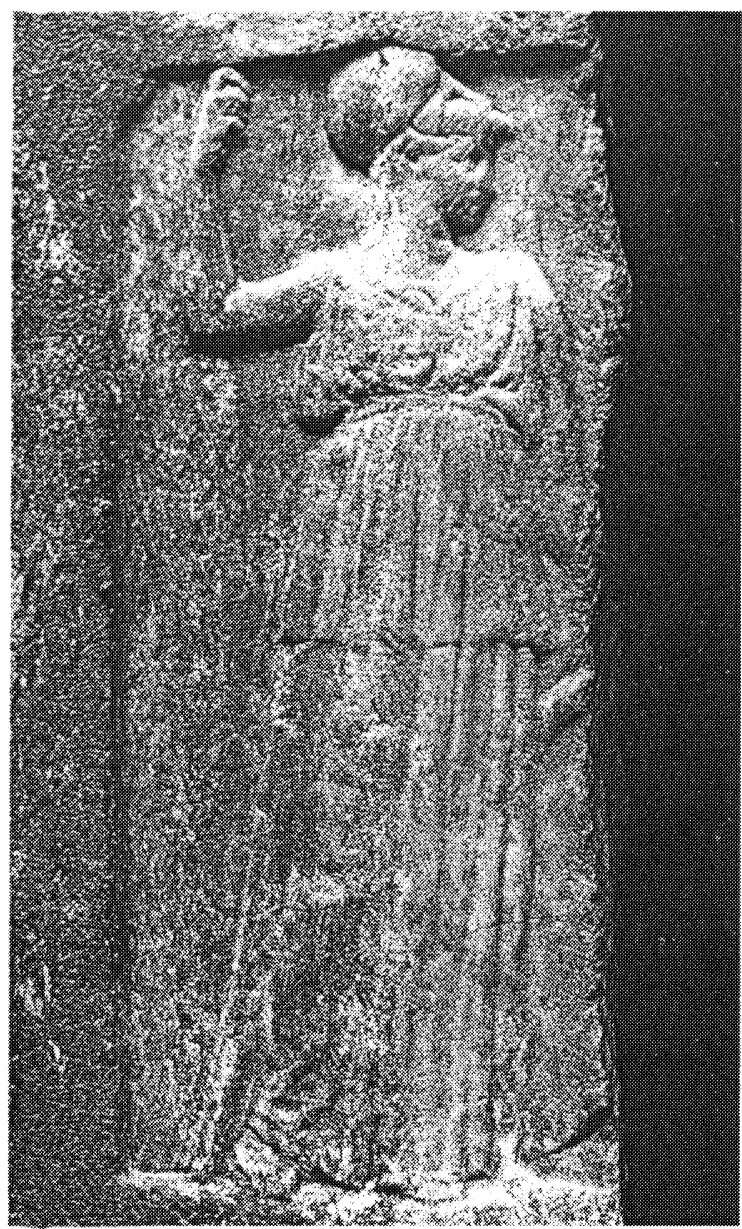

Fig. 2. - Dátail de la fig. 1 .

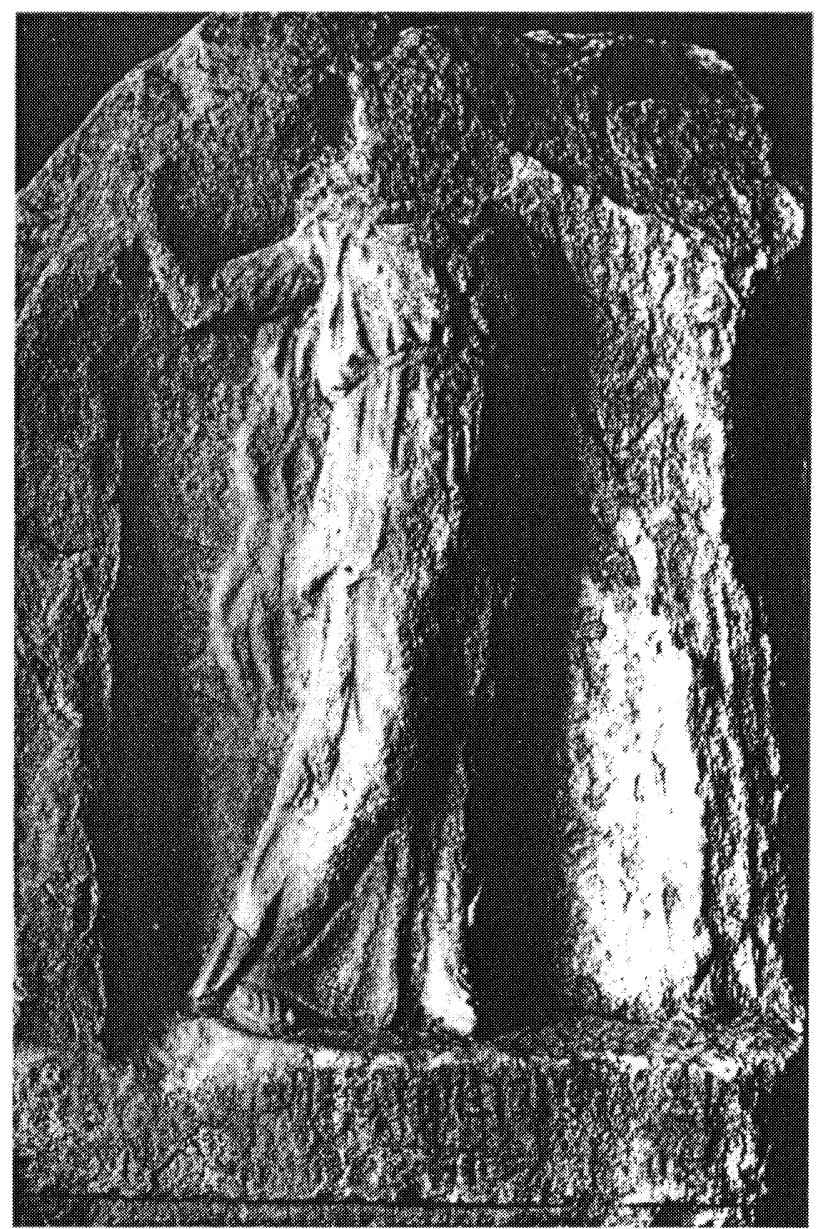

Fig, 3. - - En-tête de décret, Musce êpigraphique d'Athenes.

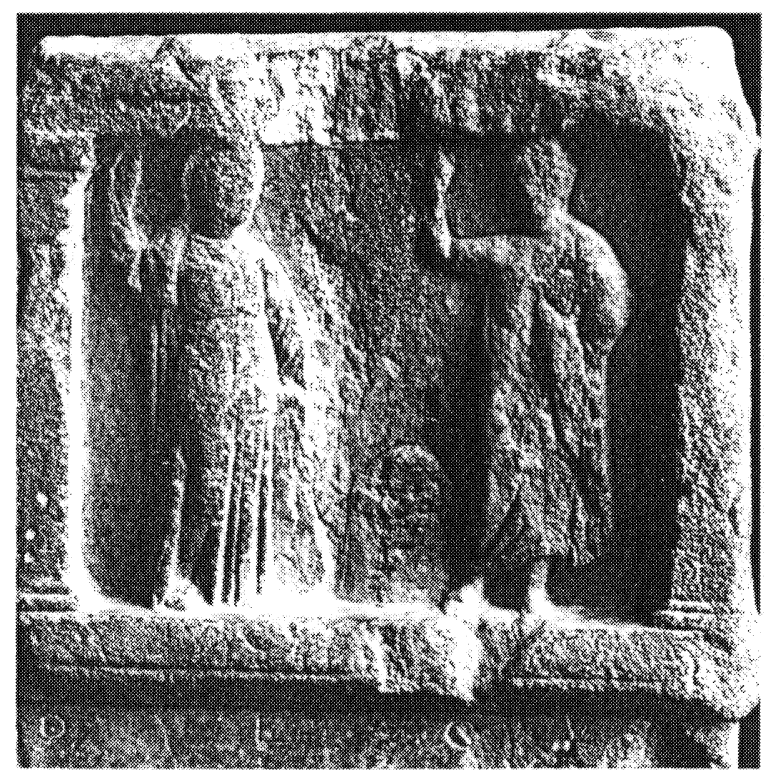

Fig. 4. - Enmete de décret, Musée de Delphes. 


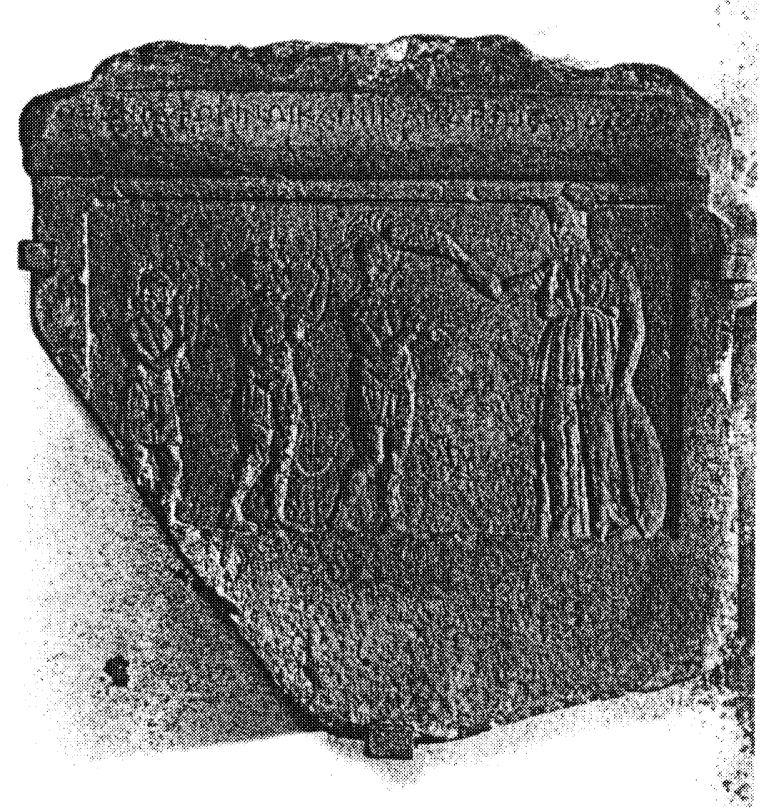

Fig. 5. - En-tête de décret, Musée Calvet, Avignon.

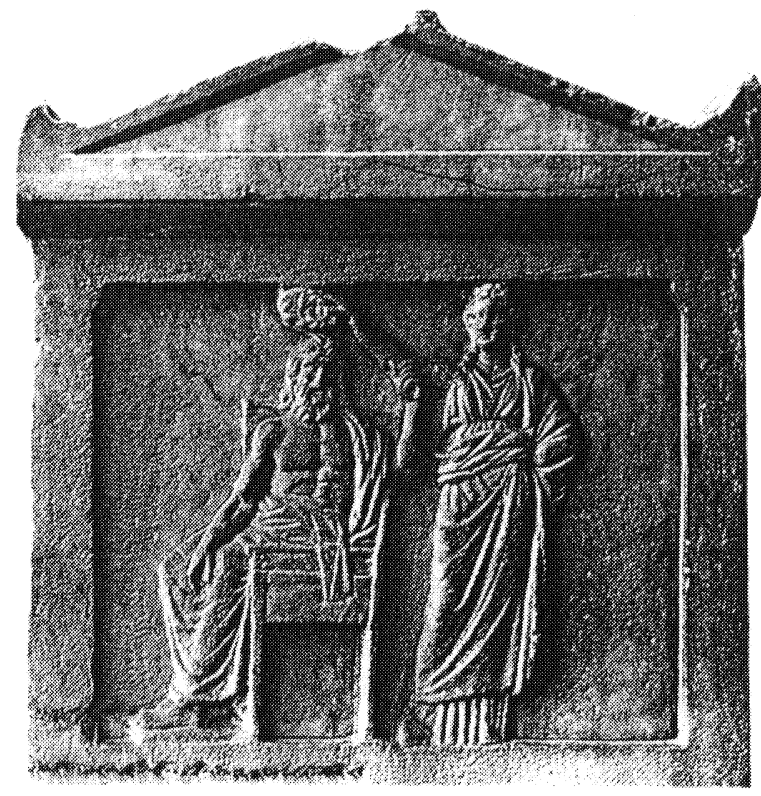

Fig. 6. - En-tête de décret, Agora d'Athènes.

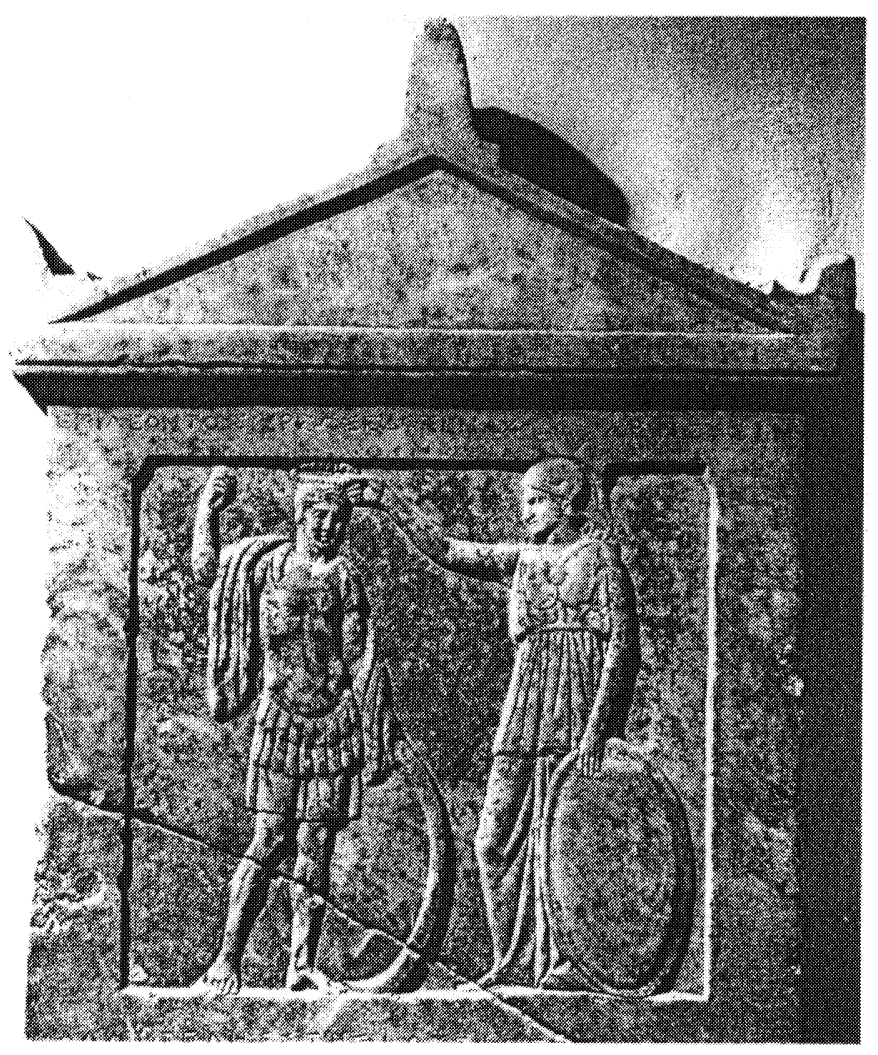

Fig. 7. - En-tête de décret, École française d'Athènes. 